\title{
Existence and uniqueness of positive solutions for the Neumann $p$-Laplacian
}

\author{
Leszek Gasiński · Nikolaos S. Papageorgiou
}

Received: 4 December 2011 / Accepted: 14 February 2012 / Published online: 4 March 2012

C The Author(s) 2012. This article is published with open access at Springerlink.com

\begin{abstract}
We consider a nonlinear Neumann problem driven by the $p$-Laplacian and with a Carathéodory reaction which satisfies only a unilateral growth restriction. Using the principal eigenvalue of an eigenvalue problem involving the Neumann $p$-Laplacian plus an indefinite potential, we produce necessary and sufficient conditions for the existence and uniqueness of positive smooth solutions.
\end{abstract}

Keywords $p$-Laplacian $\cdot$ Nonlinear strong maximum principle $\cdot$ Positive solutions . Unilateral growth restriction

Mathematics Subject Classification (2000) $\quad 35 \mathrm{~J} 65 \cdot 35 \mathrm{~J} 70 \cdot 35 \mathrm{~J} 92$

\section{Introduction}

Let $\Omega \subseteq \mathbb{R}^{N}$ be a bounded domain with a $C^{2}$-boundary $\partial \Omega$. In this paper we study the following nonlinear Neumann problem:

L. Gasiński's research has been partially supported by the Ministry of Science and Higher Education of Poland under Grants no. N201 542438 and N201 604640.

L. Gasiński $(\bowtie)$

Faculty of Mathematics and Computer Science, Jagiellonian University,

ul. Łojasiewicza 6, 30-348 Kraków, Poland

e-mail: Leszek.Gasinski@ii.uj.edu.pl

N. S. Papageorgiou

Department of Mathematics, National Technical University,

Zografou Campus, 15780 Athens, Greece

e-mail:npapg@math.ntua.gr 


$$
\left\{\begin{array}{l}
-\Delta_{p} u(z)=f(z, u(z)) \text { in } \Omega \\
\frac{\partial u}{\partial n}=0 \text { on } \partial \Omega, \quad u>0
\end{array}\right.
$$

Here $\Delta_{p}$ denotes the $p$-Laplace differential operator, defined by

$$
\Delta_{p} u=\operatorname{div}\left(\|\nabla u\|^{p-2} \nabla u\right) \quad \forall u \in W^{1, p}(\Omega),
$$

with $p \in(1,+\infty)$. Also, the reaction $f(z, \zeta)$ is a Carathéodory function, i.e., for all $\zeta \in \mathbb{R}, z \longmapsto f(z, \zeta)$ is measurable and for almost all $z \in \Omega, \zeta \longmapsto f(z, \zeta)$ is continuous.

We are interested in the existence and uniqueness of positive solutions when the nonlinearity $f(z, \cdot)$ is only unilaterally restricted (only from above). Problems like this were studied primarily in the context of semilinear (i.e., $p=2$ ) equations with Dirichlet boundary conditions. We mention the works of Amann [2], Brézis and Oswald [4], Dancer [6], de Figueiredo [7], Hess [16], Krasnoselskii [19], Laetsch [20], and Simpson and Cohen [24]. Extensions to the Dirichlet $p$-Laplacian can be found in the works of Guo [14], Guo and Webb [15] and Kamin and Veron [18], but for special classes of equations, such as logistic equations. To the best of our knowledge, there are no such results for the Neumann $p$-Laplacian. Some other existence results for Neumann $p$-Laplacian problems, but with no information on the sign of solutions can be found in Gasiński and Papageorgiou [9-11] and with some sign information on the solution (but without uniqueness) can be found in Gasiński and Papageorgiou [12,13].

As it is remarked in de Figueiredo [7], the problem of uniqueness for elliptic equations, is in general a difficult one and requires special structure on the reaction term. Our work here is closely related to that of Brézis and Oswald [4]. In fact our result is a twofold generalization of that in [4]. First, we pass from the Laplacian (semilinear equation; i.e., $p=2$ ) to the $p$-Laplacian (nonlinear equation; i.e., $p \in(1,+\infty)$ ). Second, we pass from the Dirichlet to the Neumann boundary condition. We should mention that sufficient conditions for the uniqueness of the positive solutions of the Dirichlet $p$-Laplacian were obtained by Belloni and Kawohl [3], were the authors exploited in a direct way the convexity of the energy functional $u \longmapsto \varphi(u)$ in $u^{p}$.

\section{An eigenvalue problem}

In this section we discuss the first eigenvalue of the nonlinear eigenvalue problem involving the negative Neumann $p$-Laplacian plus an indefinite potential. This quantity plays a central role in our subsequential considerations, but it is also of independent interest.

The eigenvalue problem under consideration is the following:

$$
\left\{\begin{array}{l}
-\Delta_{p} u(z)+\beta(z)|u(z)|^{p-2} u(z)=\widehat{\lambda}|u(z)|^{p-2} u(z) \text { in } \Omega \\
\frac{\partial u}{\partial n}=0 \text { on } \partial \Omega
\end{array}\right.
$$


Proposition 2.1 If $\beta \in L^{\infty}(\Omega)$, then problem (2.1) has a smallest eigenvalue $\widehat{\lambda}_{1}=$ $\widehat{\lambda}_{1}(\beta) \in \mathbb{R}$ which is simple, has a corresponding $L^{p}$-normalized eigenfunction $\widehat{u}_{1} \in$ $C^{1, \alpha}(\bar{\Omega}), 0<\alpha<1$ with $\widehat{u}_{1}(z)>0$ for all $z \in \bar{\Omega}$.

Proof Let $\xi: W^{1, p}(\Omega) \longrightarrow \mathbb{R}$ be the $C^{1}$-functional, defined by

$$
\xi(u)=\|\nabla u\|_{p}^{p}+\int_{\Omega} \beta|u|^{p} d z
$$

and let $M \subseteq W^{1, p}(\Omega)$ be the $C^{1}$-Banach manifold, defined by

$$
M=\left\{u \in W^{1, p}(\Omega):\|u\|_{p}=1\right\} .
$$

We set

$$
\widehat{\lambda}_{1}=\widehat{\lambda}_{1}(\beta)=\inf \{\xi(u): u \in M\}
$$

Because for $u \in M$, we have

$$
\left.\left.\left|\int_{\Omega} \beta\right| u\right|^{p} d z\left|\leqslant \int_{\Omega}\right| \beta|| u\right|^{p} d z \leqslant\|\beta\|_{\infty}\|u\|_{p}^{p}=\|\beta\|_{\infty}
$$

so

$$
\xi(u)=\|\nabla u\|_{p}^{p}+\int_{\Omega} \beta|u|^{p} d z \geqslant\|\nabla u\|_{p}^{p}-\|\beta\|_{\infty} \geqslant-\|\beta\|_{\infty} \quad \forall u \in M .
$$

Thus $\widehat{\lambda}_{1} \geqslant-\|\beta\|_{\infty}$. We will show that the infimum in (2.2) is realized at a $\widehat{u}_{1} \in$ $W^{1, p}(\Omega)$, with $\left\|\widehat{u}_{1}\right\|_{p}=1$. To this end, let $\left\{u_{n}\right\}_{n} \geqslant 1 \subseteq M$ be a minimizing sequence, i.e.,

$$
\xi\left(u_{n}\right) \longrightarrow \widehat{\lambda}_{1}
$$

Clearly the sequence $\left\{u_{n}\right\}_{n} \geqslant 1 \subseteq W^{1, p}(\Omega)$ is bounded and so by passing to a suitable subsequence if necessary, we may assume that

$$
\begin{array}{ll}
u_{n} \stackrel{w}{\longrightarrow} \widehat{u}_{1} \text { in } W^{1, p}(\Omega), \\
u_{n} \longrightarrow \widehat{u}_{1} \quad \text { in } L^{p}(\Omega) .
\end{array}
$$

From (2.3) and (2.3), we have

$$
\left\|\nabla \widehat{u}_{1}\right\|_{p}^{p} \leqslant \liminf _{n \rightarrow+\infty}\left\|\nabla u_{n}\right\|_{p}^{p} \text { and } \lim _{n \rightarrow+\infty} \int_{\Omega} \beta\left|u_{n}\right|^{p} d z=\int_{\Omega} \beta\left|\widehat{u}_{1}\right|^{p} d z
$$


so

$$
\xi\left(\widehat{u}_{1}\right) \leqslant \widehat{\lambda}_{1} \text {. }
$$

It is clear from (2.3) that $\left\|\widehat{u}_{1}\right\|_{p}=1$, i.e., $\widehat{u}_{1} \in M$. Hence $\xi\left(\widehat{u}_{1}\right)=\widehat{\lambda}_{1}$.

The Lagrange multiplier rule (see, e.g., Papageorgiou and Kyritsi [23, p. 76]) implies that $\widehat{\lambda}_{1}$ is an eigenvalue of problem (2.1), with the corresponding eigenfunction $\widehat{u}_{1} \in$ $W^{1, p}(\Omega)$. Using the Moser iteration technique, we show that $\widehat{u}_{1} \in L^{\infty}(\Omega)$ (see, e.g., $\mathrm{Hu}$ and Papageorgiou [17]) and the nonlinear regularity theorem of Lieberman [21], implies that $\widehat{u}_{1} \in C^{1, \alpha}(\bar{\Omega})$ for some $\alpha \in(0,1)$. Moreover, since

$$
\xi(|u|)=\xi(u) \quad \forall u \in M,
$$

we infer that $\widehat{u}_{1}$ does not change sign and we may assume that $\widehat{u}_{1} \geqslant 0$. Invoking the nonlinear maximum principle of Vázquez [25], we conclude that

$$
\widehat{u}_{1}(z)>0 \quad \forall z \in \bar{\Omega} \text {. }
$$

Next, we show the simplicity of $\widehat{\lambda}_{1}$. So, let $\widehat{v}_{1} \in W^{1, p}(\Omega)$ be another eigenfunction corresponding to $\widehat{\lambda}_{1}$. As above, we show that $\widehat{v}_{1} \in C^{1}(\bar{\Omega})$ and $\widehat{v}_{1}(z)>0$ for all $z \in \bar{\Omega}$. We introduce

$$
R\left(\widehat{u}_{1}, \widehat{v}_{1}\right)(z)=\left\|\nabla \widehat{u}_{1}(z)\right\|^{p}-\left\|\nabla \widehat{v}_{1}(z)\right\|^{p-2}\left(\nabla \widehat{v}_{1}(z), \nabla\left(\frac{\widehat{u}_{1}(z)^{p}}{\widehat{v}_{1}(z)^{p-1}}\right)\right)_{\mathbb{R}^{N}}
$$

From the generalized Picone identity of Allegretto and Huang [1] and the nonlinear Green's identity (see Casas and Fernández [5]), we have

$$
\begin{aligned}
0 & \leqslant \int_{\Omega} R\left(\widehat{u}_{1}, \widehat{v}_{1}\right) d z \\
& =\int_{\Omega}\left[\left\|\nabla \widehat{u}_{1}\right\|^{p}-\left\|\nabla \widehat{v}_{1}\right\|^{p-2}\left(\nabla \widehat{v}_{1}, \nabla\left(\frac{\widehat{u}_{1}^{p}}{\widehat{v}_{1}^{p-1}}\right)\right)_{\mathbb{R}^{N}}\right] d z \\
& =\int_{\Omega}\left[\left\|\nabla \widehat{u}_{1}\right\|^{p}+\Delta_{p} \widehat{v}_{1}\left(\frac{\widehat{u}_{1}^{p}}{\widehat{v}_{1}^{p-1}}\right)\right] d z \\
& =\int_{\Omega}\left[\left\|\nabla \widehat{u}_{1}\right\|^{p}+\left(\beta(z)-\widehat{\lambda}_{1}\right) \widehat{v}_{1}^{p-1} \frac{\widehat{u}_{1}^{p}}{\widehat{v}_{1}^{p-1}}\right] d z \\
& =\int_{\Omega}\left[\left\|\nabla \widehat{u}_{1}\right\|^{p}+\beta \widehat{u}_{1}^{p}\right] d z-\widehat{\lambda}_{1}\left\|\widehat{u}_{1}\right\|_{p}^{p} \\
& =\xi\left(\widehat{u}_{1}\right)-\widehat{\lambda}_{1}\left\|\widehat{u}_{1}\right\|_{p}^{p}=0,
\end{aligned}
$$


so

$$
\int_{\Omega} R\left(\widehat{u}_{1}, \widehat{v}_{1}\right) d z=0
$$

and thus

$$
R\left(\widehat{u}_{1}, \widehat{v}_{1}\right)=0 \quad \forall z \in \bar{\Omega},
$$

so finally $\widehat{u}_{1}=k \widehat{v}_{1}$ for some $k>0$ (see Allegretto and Huang [1]). This proves that $\widehat{\lambda}_{1}$ is simple (i.e., it is a principal eigenvalue).

From the above proof, we have

$$
\begin{aligned}
\widehat{\lambda}_{1}(\beta) & =\inf \left\{\int_{\Omega}\|\nabla u\|^{p} d z+\int_{\Omega} \beta|u|^{p} d z: u \in W^{1, p}(\Omega),\|u\|_{p}=1\right\} \\
& =\inf \left\{\int_{\Omega}\|\nabla u\|^{p} d z+\int_{\{u \neq 0\}} \beta|u|^{p} d z: u \in W^{1, p}(\Omega),\|u\|_{p}=1\right\} .
\end{aligned}
$$

Note that in the second infimum in (2.6), the integral $\int_{\{u \neq 0\}} \beta|u|^{p} d z$ makes sense even when $\beta$ is only a measurable function and there exists $\widehat{c}>0$, such that

$$
\beta(z) \leqslant \widehat{c} \text { for almost all } z \in \Omega
$$

or

$$
\beta(z) \geqslant-\widehat{c} \text { for almost all } z \in \Omega \text {. }
$$

In the first case $\widehat{\lambda}_{1}(\beta) \in[-\infty,+\infty)$ and in the second case $\widehat{\lambda}_{1}(\beta) \in(-\infty,+\infty]$.

In what follows by $A: W^{1, p}(\Omega) \longrightarrow W^{1, p}(\Omega)^{*}$ we denote the nonlinear map, defined by

$$
\langle A(u), y\rangle=\int_{\Omega}\|\nabla u\|^{p-2}(\nabla u, \nabla y)_{\mathbb{R}^{N}} d z \quad \forall u, y \in W^{1, p}(\Omega) .
$$

This map is continuous and maximal monotone (see [8] or [23]).

\section{Existence of positive solutions}

In this section we prove the existence of a positive smooth solution. The hypotheses on the reaction $f$ are the following:

$H_{f}: f: \Omega \times \mathbb{R} \longrightarrow \mathbb{R}$ is a Carathéodory function, such that 
(i) for all $\zeta \geqslant 0, f(\cdot, \zeta) \in L^{\infty}(\Omega)$ and there exists $c>0$, such that

$$
f(z, \zeta) \leqslant c\left(1+\zeta^{p-1}\right) \text { for almost all } z \in \Omega, \text { all } \zeta \geqslant 0
$$

(ii) for almost all $z \in \Omega$, the function $\zeta \longrightarrow \frac{f(z, \zeta)}{\zeta^{p-1}}$ is strictly decreasing on $(0,+\infty)$;

(iii) if $\eta(z)=\lim _{\zeta \rightarrow+\infty} \frac{f(z, \zeta)}{\zeta^{p-1}}$, then $\widehat{\lambda}_{1}(-\eta)>0$;

(iv) if $\eta_{0}(z)=\lim _{\zeta \rightarrow 0^{+}} \frac{f(z, \zeta)}{\zeta^{p-1}}$, then $\widehat{\lambda}_{1}\left(-\eta_{0}\right)<0$.

Remark 3.1 Since we are looking for positive solutions and hypotheses $H_{f}$ concern only the positive semiaxis $\mathbb{R}_{+}=[0,+\infty)$, by truncating if necessary, we may (and will) assume that

$$
f(z, \zeta)=f(z, 0) \text { for almost all } \zeta \leqslant 0
$$

Note that $H_{f}$ (i) is a unilateral growth condition. Hypothesis $H_{f}$ (ii) implies that both functions $\eta$ and $\eta_{0}$ are measurable. Moreover, we have

$$
\frac{f(z, \zeta)}{\zeta^{p-1}} \leqslant f(z, 1) \leqslant\|f(\cdot, 1)\|_{\infty}=\widehat{c} \text { for almost all } z \in \Omega, \text { all } \zeta \geqslant 1,
$$

so

$$
\eta(z) \leqslant \widehat{c} \text { for almost all } z \in \Omega
$$

and thus

$$
\widehat{\lambda}_{1}(-\eta) \in(-\infty,+\infty]
$$

Similarly, we have

$$
\frac{f(z, \zeta)}{\zeta^{p-1}} \geqslant f(z, 1) \geqslant-\|f(\cdot, 1)\|_{\infty}=-\widehat{c} \text { for almost all } z \in \Omega \text {, all } \zeta \in(0,1]
$$

so

$$
\eta_{0}(z) \geqslant-\widehat{c} \text { for almost all } z \in \Omega
$$

and thus

$$
\widehat{\lambda}_{1}\left(-\eta_{0}\right) \in[-\infty,+\infty) .
$$

If $\eta, \eta_{0} \in L^{\infty}(\Omega)$, then $\widehat{\lambda}_{1}(-\eta), \widehat{\lambda}\left(-\eta_{0}\right) \in \mathbb{R}$ and are the principal eigenvalues of (2.1) when $\beta=-\eta$ and $\beta=-\eta_{0}$ respectively. If $f(z, \zeta)=f(\zeta)$ (autonomous case), then hypotheses $H_{f}$ (iii) and $H_{f}$ (iv) are equivalent to saying that

$$
\eta<\widehat{\lambda}_{1}=0<\eta_{0}
$$


(recall that the first eigenvalue of the negative Neumann $p$-Laplacian (i.e., problem (2.1) with $\beta \equiv 0$ ) is zero).

Example 3.2 Let

$$
f(\zeta)=\lambda\left(\zeta^{p-1}-\zeta^{q-1}\right) \quad \forall \zeta \geqslant 0
$$

with $1<p<q, \lambda>0$. Then $f$ satisfies hypotheses $H_{f}$. This function corresponds to the equidiffusive $p$-logistic equation and $\eta_{0}=\lambda>0, \eta=-\infty$. More generally, let

$$
f(\zeta)= \begin{cases}\zeta^{p-1}-\zeta^{q-1} & \text { if } \zeta \in[0,1] \\ \zeta^{p-1}-e^{\zeta-1} & \text { if } \zeta \geqslant 1\end{cases}
$$

with $1<p \leqslant q$. Note that this $f$ has no polynomial growth restriction from below.

We introduce the following truncation-perturbation of $f$ :

$$
g(z, \zeta)= \begin{cases}f(z, 0) & \text { if } \zeta \leqslant 0 \\ f(z, \zeta)+\zeta^{p-1} & \text { if } \zeta>0\end{cases}
$$

This is a Carathéodory function. We set

$$
F(z, \zeta)=\int_{0}^{\zeta} f(z, s) d s \text { and } G(z, \zeta)=\int_{0}^{\zeta} g(z, s) d s
$$

Note that hypothesis $H_{f}$ (i) and (3.1) imply that

$$
G(z, \zeta) \leqslant c_{1}\left(1+\zeta^{p}\right) \text { for almost all } z \in \Omega, \text { all } \zeta \in \mathbb{R}
$$

and some $c_{1}>0$. Because of (3.2), we see that we can introduce the functional $\widehat{\varphi}: W^{1, p}(\Omega) \longrightarrow \overline{\mathbb{R}}=\mathbb{R} \cup\{\infty\}$, defined by

$$
\widehat{\varphi}=\frac{1}{p}\|\nabla u\|_{p}^{p}+\frac{1}{p}\|u\|_{p}^{p}-\int_{\Omega} G(z, u) d z \quad \forall u \in W^{1, p}(\Omega) .
$$

Proposition 3.3 If hypotheses $H_{f}$ hold, then $\widehat{\varphi}$ is coercive, i.e., $\widehat{\varphi}(u) \longrightarrow+\infty$ as $\|u\| \rightarrow+\infty$.

Proof We argue by contradiction. So, suppose that we can find a sequence $\left\{u_{n}\right\}_{n} \geqslant 1 \subseteq$ $W^{1, p}(\Omega)$, such that

$$
\left\|u_{n}\right\| \longrightarrow+\infty \text { and } \widehat{\varphi}\left(u_{n}\right) \leqslant M_{1} \quad \forall n \geqslant 1,
$$

for some $M_{1}>0$. We have

$$
\frac{1}{p}\left(\left\|\nabla u_{n}\right\|_{p}^{p}+\left\|u_{n}\right\|_{p}^{p}\right) \leqslant c_{2}\left(1+\left\|u_{n}\right\|_{p}^{p}\right) \quad \forall n \geqslant 1
$$


for some $c_{2}>0$ (see (3.2) and (3.3)).

It is clear from (3.3) and (3.4) that $\left\|u_{n}\right\|_{p} \longrightarrow+\infty$. We set

$$
y_{n}=\frac{u_{n}}{\left\|u_{n}\right\|_{p}} \quad \forall n \geqslant 1
$$

Then

$$
\left\|y_{n}\right\|_{p}=1 \quad \forall n \geqslant 1
$$

and from (3.4), we have

$$
\frac{1}{p}\left(\left\|\nabla y_{n}\right\|_{p}^{p}+\left\|y_{n}\right\|_{p}^{p}\right) \leqslant c_{2}\left(\frac{1}{\left\|u_{n}\right\|_{p}^{p}}+1\right)
$$

so the sequence $\left\{y_{n}\right\}_{n} \geqslant 1 \subseteq W^{1, p}(\Omega)$ is bounded.

So, passing to a subsequence if necessary, we may assume that

$$
y_{n} \stackrel{w}{\longrightarrow} y \text { in } W^{1, p}(\Omega),
$$

$$
y_{n} \longrightarrow y \text { in } L^{p}(\Omega)
$$

hence $\|y\|_{p}=1$. We have

$$
\begin{aligned}
& \frac{1}{p}\left(\left\|\nabla y_{n}\right\|_{p}^{p}+\left\|y_{n}\right\|_{p}^{p}\right) \leqslant \frac{M_{1}}{\left\|u_{n}\right\|_{p}^{p}}+\int_{\Omega} \frac{G\left(z, u_{n}\right)}{\left\|u_{n}\right\|_{p}^{p}} d z \\
& \quad \leqslant \frac{M_{1}}{\left\|u_{n}\right\|_{p}^{p}}+\int_{\left\{u_{n}>0\right\}}\left(\frac{F\left(z, u_{n}\right)}{\left\|u_{n}\right\|_{p}^{p}}+\frac{1}{p} y_{n}^{p}\right) d z+\int_{\left\{u_{n} \leqslant 0\right\}} \frac{f(z, 0) u_{n}}{\left\|u_{n}\right\|_{p}^{p}} d z
\end{aligned}
$$

(see (3.1)). Note that

$$
\frac{f(z, \zeta)}{\zeta^{p-1}} \geqslant f(z, 1) \text { for almost all } z \in \Omega, \text { all } \zeta \in(0,1]
$$

(see hypothesis $H_{f}(\mathrm{ii})$ ). Hence

$f(z, \zeta) \geqslant f(z, 1) \zeta^{p-1} \geqslant-\|f(\cdot, 1)\|_{\infty} \zeta^{p-1}$ for almost all $z \in \Omega$, all $\zeta \in(0,1]$

So, it follows that

$$
f(z, 0) \geqslant 0 \text { for almost all } z \in \Omega \text {. }
$$


Then

$$
\int_{\left\{u_{n} \leqslant 0\right\}} \frac{f(z, 0) u_{n}}{\left\|u_{n}\right\|_{p}^{p}} d z \leqslant 0 \quad \forall n \geqslant 1
$$

Using (3.9) in (3.8), we obtain

$$
\frac{1}{p}\left(\left\|\nabla y_{n}\right\|_{p}^{p}+\left\|y_{n}\right\|_{p}^{p}\right) \leqslant \frac{M_{1}}{\left\|u_{n}\right\|_{p}^{p}}+\frac{1}{p}\left\|y_{n}^{+}\right\|_{p}^{p}+\int_{\Omega} \frac{F\left(z, u_{n}^{+}\right)}{\left\|u_{n}\right\|_{p}^{p}} d z \quad \forall n \geqslant 1
$$

Suppose that the sequence $\left\{u_{n}^{+}\right\}_{n} \geqslant 1 \subseteq L^{p}(\Omega)$ is bounded. Then $y \leqslant 0$. From hypothesis $H_{f}(\mathrm{i})$, we have

$$
F(z, \zeta) \leqslant c_{3}\left(1+\zeta^{p}\right) \text { for almost all } z \in \Omega, \text { all } \zeta \geqslant 0
$$

and some $c_{3}>0$. Then using (3.11), we have

$$
\int_{\Omega} \frac{F\left(z, u_{n}^{+}\right)}{\left\|u_{n}\right\|_{p}^{p}} d z \leqslant \frac{c_{3}|\Omega|_{N}}{\left\|u_{n}\right\|_{p}^{p}}+c_{3}\left\|y_{n}^{+}\right\|_{p}^{p}
$$

$\left(|\cdot|_{N}\right.$ denotes the Lebesgue measure on $\left.\mathbb{R}^{N}\right)$, so

$$
\limsup _{n \rightarrow+\infty} \int_{\Omega} \frac{F\left(z, u_{n}^{+}\right)}{\left\|u_{n}\right\|_{p}^{p}} d z \leqslant 0
$$

(see (3.7) and recall that $y \leqslant 0$ ). So, if in (3.10) we pass to the limit as $n \rightarrow+\infty$, we obtain

$$
\frac{1}{p}\left(\|\nabla y\|_{p}^{p}+\|y\|_{p}^{p}\right) \leqslant 0
$$

so $y=0$, which contradicts (3.5) and (3.7).

Therefore we may assume that $\left\|u_{n}^{+}\right\|_{p} \longrightarrow+\infty$. From the inequality in (3.3), we have

$$
\frac{1}{p}\left\|\nabla y_{n}^{+}\right\|_{p}^{p} \leqslant \frac{M_{1}}{\left\|u_{n}^{+}\right\|_{p}^{p}}+\int_{\Omega} \frac{F\left(z, u_{n}^{+}\right)}{\left\|u_{n}^{+}\right\|_{p}^{p}} d z \quad \forall n \geqslant 1
$$


(see (3.9)). We have

$$
\begin{aligned}
\int_{\Omega} \frac{F\left(z, u_{n}^{+}\right)}{\left\|u_{n}^{+}\right\|_{p}^{p}} d z= & \int_{\left\{y^{+}=0\right\}} \frac{F\left(z, u_{n}^{+}\right)}{\left\|u_{n}^{+}\right\|_{p}^{p}} d z \\
& +\int_{\{y>0\} \cap\left\{y_{n}>0\right\}} \frac{F\left(z, u_{n}^{+}\right)}{\left(u_{n}^{+}\right)^{p}}\left(y_{n}^{+}\right)^{p} d z \quad \forall n \geqslant 1 .
\end{aligned}
$$

Since

$$
y_{n}^{+} \longrightarrow y^{+} \text {in } L^{p}(\Omega)
$$

(see (3.7)), by passing to a further subsequence if necessary, we may also assume that

$$
y_{n}^{+}(z) \longrightarrow y^{+}(z) \text { for almost all } z \in \Omega \text {. }
$$

From (3.11), we have

$$
\left|\int_{\left\{y^{+}=0\right\}} \frac{F\left(z, u_{n}^{+}\right)}{\left\|u_{n}^{+}\right\|_{p}^{p}} d z\right| \leqslant c_{3} \int_{\left\{y^{+}=0\right\}}\left(\frac{1}{\left\|u_{n}^{+}\right\|_{p}^{p}}+\left(y_{n}^{+}\right)^{p}\right) d z \longrightarrow 0 .
$$

Note that

$$
u_{n}^{+}(z) \longrightarrow+\infty \text { almost everywhere on }\left\{y^{+}>0\right\}
$$

and

$$
\chi_{\{y>0\} \cap\left\{y_{n}>0\right\}}(z) \longrightarrow \chi_{\{y>0\}}(z) \text { almost everywhere in } \Omega \text {. }
$$

Moreover, we claim that

$$
\limsup _{\zeta \rightarrow+\infty} \frac{F(z, \zeta)}{\zeta^{p}} \leqslant \frac{1}{p} \eta(z) \text { for almost all } z \in \Omega \text {. }
$$

Indeed, first let $z \in\{\eta>-\infty\} \backslash D$, with $|D|_{N}=0$ be such that

$$
\frac{f(z, \zeta)}{\zeta^{p-1}} \longrightarrow \eta(z) \text { as } \zeta \rightarrow+\infty
$$

(see hypotheses $H_{f}$ (ii) and (iii)). For a given $\varepsilon>0$, we can find $M_{2}=M_{2}(\varepsilon, z)>0$, such that

$$
f(z, \zeta) \leqslant(\eta(z)+\varepsilon) \zeta^{p-1} \quad \forall \zeta \geqslant M_{2}
$$


so

$$
F(z, \zeta) \leqslant \frac{1}{p}(\eta(z)+\varepsilon) \zeta^{p} \quad \forall \zeta \geqslant M_{2}
$$

thus

$$
\frac{F(z, \zeta)}{\zeta^{p}} \leqslant \frac{1}{p}(\eta(z)+\varepsilon) \quad \forall \zeta \geqslant M_{2}
$$

and so

$$
\limsup _{\zeta \rightarrow+\infty} \frac{F(z, \zeta)}{\zeta^{p}} \leqslant \frac{1}{p}(\eta(z)+\varepsilon)
$$

Since $\varepsilon>0$ was arbitrary, we let $\varepsilon \searrow 0$ to conclude that

$$
\limsup _{\zeta \rightarrow+\infty} \frac{F(z, \zeta)}{\zeta^{p}} \leqslant \frac{1}{p} \eta(z) \text { for almost all } z \in\{\eta>-\infty\}
$$

If $z \in\{\eta=-\infty\} \backslash D$, with $|D|_{N}=0$ is such that

$$
\frac{f(z, \zeta)}{\zeta^{p-1}} \longrightarrow-\infty=\eta(z) \text { as } \zeta \rightarrow+\infty
$$

then for every $\xi>0$, we can find $M_{3}=M_{3}(\xi, z)>0$, such that

$$
f(z, \zeta) \leqslant-\xi \zeta^{p-1} \quad \forall \zeta \geqslant M_{3}
$$

so

$$
\frac{F(z, \zeta)}{\zeta^{p}} \leqslant-\frac{\xi}{p} \quad \forall \zeta \geqslant M_{3}
$$

and thus

$$
\limsup _{\zeta \rightarrow+\infty} \frac{F(z, \zeta)}{\zeta^{p}} \leqslant-\frac{\xi}{p}
$$

Since $\xi>0$ was arbitrary, we let $\xi \rightarrow+\infty$ to conclude that

$$
\lim _{\zeta \rightarrow+\infty} \frac{F(z, \zeta)}{\zeta^{p}}=-\infty \text { for almost all } z \in\{\eta=-\infty\}
$$

Therefore, finally we have proved (3.18). 
Using Fatou's lemma in (3.18) (which is legitimate because of (3.11)) as well as (3.16), (3.17) and (3.14), we have

$$
\limsup _{n \rightarrow+\infty} \int_{\{y>0\} \cap\left\{y_{n}>0\right\}} \frac{F\left(z, u_{n}^{+}\right)}{\left(u_{n}^{+}\right)^{p}}\left(y_{n}^{+}\right)^{p} d z \leqslant \frac{1}{p} \int_{\{y>0\}} \eta y^{p} d z=\frac{1}{p} \int_{\left\{y^{+} \neq 0\right\}} \eta\left(y^{+}\right)^{p} d z .
$$

Hence, if in (3.13) we pass to the limit as $n \rightarrow+\infty$ and use (3.15) and (3.19), we obtain

$$
\limsup _{n \rightarrow+\infty} \int_{\Omega} \frac{F\left(z, u_{n}^{+}\right)}{\left\|u_{n}^{+}\right\|^{p}} d z \leqslant \frac{1}{p} \int_{\left\{y^{+} \neq 0\right\}} \eta\left(y^{+}\right)^{p} d z
$$

Returning to (3.12), taking limits as $n \rightarrow+\infty$ and using (3.6) and (3.20), we have

$$
\left\|\nabla y^{+}\right\|_{p}^{p} \leqslant \frac{1}{p} \int_{\left\{y^{+} \neq 0\right\}} \eta\left(y^{+}\right)^{p} d z
$$

If $y^{+}=0$, then from (3.10), we have

$$
\frac{1}{p}\left(\left\|\nabla y^{-}\right\|_{p}^{p}+\left\|y^{-}\right\|_{p}^{p}\right) \leqslant 0
$$

so $y^{-}=0$, i.e., $y=0$ which contradicts (3.6).

So $y^{+} \neq 0$ and then from (3.21), it follows that

$$
\widehat{\lambda}_{1}(-\eta) \leqslant 0
$$

(see (2.6)), which contradicts hypothesis $H_{f}$ (iii). This proves that $\widehat{\varphi}$ is coercive.

Proposition 3.4 If hypotheses $H_{f}$ hold, then $\widehat{\varphi}$ is sequentially weakly lower semicontinuous.

Proof From the expression of $\widehat{\varphi}$ and since the norm in a Banach space is sequentially weakly lower semicontinuous, it suffices to show that the integral functional $\psi: W^{1, p}(\Omega) \longrightarrow \overline{\mathbb{R}}=\mathbb{R} \cup\{+\infty\}$, defined by

$$
\psi(u)=-\int_{\Omega} G(z, u) d z
$$

is sequentially weakly lower semicontinuous. To this end, we need to show that for every $\lambda \in \mathbb{R}$, the sublevel set

$$
L_{\lambda}=\left\{u \in W^{1, p}(\Omega): \psi(u) \leqslant \lambda\right\}
$$


is sequentially weakly closed. To this end, let $\left\{u_{n}\right\}_{n} \geqslant 1 \subseteq L_{\lambda}$ and assume that

$$
u_{n} \stackrel{w}{\longrightarrow} u \text { in } W^{1, p}(\Omega) .
$$

Then

$$
u_{n} \longrightarrow u \text { in } L^{p}(\Omega)
$$

(by the Sobolev embedding theorem) and since $L^{p}(\Omega)$ is a Banach lattice, we also have that

$$
u_{n}^{ \pm} \longrightarrow u^{ \pm} \text {in } L^{p}(\Omega)
$$

We may also assume that

$$
u_{n}^{ \pm}(z) \longrightarrow u^{ \pm}(z) \text { almost everywhere in } \Omega
$$

We have

$$
\lambda \geqslant-\int_{\Omega} G\left(z, u_{n}\right) d z=-\int_{\Omega} F\left(z, u_{n}^{+}\right) d z-\frac{1}{p}\left\|u_{n}^{+}\right\|_{p}^{p}-\int_{\Omega} f(z, 0)\left(-u_{n}^{-}\right) d z .
$$

Note that

$$
\frac{1}{p}\left\|u_{n}^{+}\right\|_{p}^{p} \longrightarrow \frac{1}{p}\left\|u^{+}\right\|_{p}^{p}
$$

and

$$
\int_{\Omega} f(z, 0)\left(-u_{n}^{-}\right) d z \rightarrow \int_{\Omega} f(z, 0)\left(-u^{-}\right) d z
$$

(see (3.22)). Also, from (3.23) and Fatou's lemma, we have

$$
\liminf _{n \rightarrow+\infty}\left(-\int_{\Omega} F\left(z, u_{n}^{+}\right) d z\right)=-\limsup _{n \rightarrow+\infty} \int_{\Omega} F\left(z, u_{n}^{+}\right) d z \geqslant-\int_{\Omega} F\left(z, u^{+}\right) d z
$$

Then, from (3.24) and using (3.25) and (3.27), in the limit as $n \rightarrow+\infty$, we have

$$
\lambda \geqslant-\int_{\Omega} F\left(z, u^{+}\right) d z-\frac{1}{p}\left\|u^{+}\right\|_{p}^{p}-\int_{\Omega} f(z, 0)\left(-u^{-}\right) d z=-\int_{\Omega} G(z, u) d z,
$$

so $u \in L_{\lambda}$ and so $\psi$ is sequentially weakly lower semicontinuous. 
Now we are ready to establish the existence of positive solutions.

Proposition 3.5 It hypotheses $H_{f}$ hold, then problem (1.1) has a positive solution $u_{0} \in C^{1}(\bar{\Omega})$ with $u_{0}(z)>0$ for all $z \in \bar{\Omega}$.

Proof Propositions 3.3, 3.4 and the Weierstrass theorem, imply that we can find $u_{0} \in$ $W^{1, p}(\Omega)$, such that

$$
\widehat{\varphi}\left(u_{0}\right)=\inf \left\{\widehat{\varphi}(u): u \in W^{1, p}(\Omega)\right\}=\widehat{m} .
$$

Claim 1. $u_{0} \geqslant 0, u_{0} \neq 0$.

Note that, if $u_{0}^{-} \neq 0$, then

$$
\begin{aligned}
\widehat{\varphi}\left(u_{0}^{+}\right) & =\frac{1}{p}\left\|\nabla u_{0}^{+}\right\|_{p}^{p}-\int_{\Omega} F\left(z, u_{0}^{+}\right) d z \\
& <\frac{1}{p}\left\|\nabla u_{0}\right\|_{p}^{p}+\frac{1}{p}\left\|u_{0}^{-}\right\|_{p}^{p}-\int_{\Omega} F\left(z, u_{0}^{+}\right) d z-\int_{\Omega} f(z, 0)\left(-u_{0}^{-}\right) d z \\
& =\widehat{\varphi}\left(u_{0}\right)
\end{aligned}
$$

(see (3.6) and recall that $f(z, 0) \geqslant 0$ for almost all $z \in \Omega$ ), which contradicts (3.28). Therefore $u_{0} \geqslant 0$.

Next we show that $u_{0} \neq 0$. By hypothesis $H_{f}$ (iv) and (2.6), we see that we can find $u \in W^{1, p}(\Omega)$, such that

$$
\|\nabla u\|_{p}^{p}-\int_{\{u \neq 0\}} \eta_{0}|u|^{p} d z<0,
$$

with $\|u\|_{p}=1$. Replacing $u$ with $|u| \in W^{1, p}(\Omega)$ if necessary, we may assume that $u \geqslant 0, u \neq 0$. Let $\left\{u_{n}\right\}_{n \geqslant 1} \subseteq C^{1}(\bar{\Omega})$ be a sequence, such that

$$
u_{n} \longrightarrow u \text { in } W^{1, p}(\Omega)
$$

(see e.g., Gasiński and Papageorgiou [8, p. 189]). Since

$$
u_{n}^{+} \longrightarrow u^{+}=u \text { in } W^{1, p}(\Omega),
$$

we may assume that $u_{n} \geqslant 0$ for all $n \geqslant 1$. Let us set

$$
\widehat{u}_{n}=\min \left\{u, u_{n}\right\} \in W^{1, p}(\Omega) \cap L^{\infty}(\Omega) \quad \forall n \geqslant 1 .
$$

Then

$$
\widehat{u}_{n} \longrightarrow u \text { in } W^{1, p}(\Omega)
$$


(see e.g., Gasiński and Papageorgiou [8, p. 198]). We may also assume that

$$
\widehat{u}_{n}(z) \longrightarrow u(z) \text { for almost all } z \in \Omega \text {. }
$$

By virtue of hypothesis $H_{f}$ (iv), we have

$$
\eta_{0}(z) \geqslant f(z, 1) \geqslant-\|f(\cdot, 1)\|_{\infty} \text { for almost all } z \in \Omega
$$

so

$$
\eta_{0}(z) \widehat{u}_{n}(z)^{p} \chi_{\left\{u_{n} \neq 0\right\}}(z) \geqslant-\|f(\cdot, 1)\|_{\infty} u(z)^{p} \quad \text { for almost all } z \in \Omega \text {. }
$$

Note that $\|f(\cdot, 1)\|_{\infty} u^{p} \in L^{1}(\Omega)$. Also, we have

$$
\eta_{0}(z) \widehat{u}_{n}(z)^{p} \chi_{\left\{u_{n} \neq 0\right\}}(z) \longrightarrow \eta_{0}(z) u(z)^{p} \chi_{\{u \neq 0\}}(z) \text { for almost all } z \in \Omega
$$

From (3.30), (3.31) and Fatou's lemma, we have

$$
\liminf _{n \rightarrow+\infty} \int_{\left\{u_{n} \neq 0\right\}} \eta_{0} \widehat{u}_{n}^{p} d z \geqslant \int_{\{u \neq 0\}} \eta_{0} u^{p} d z
$$

Since $\widehat{u}_{n} \longrightarrow u$ in $W^{1, p}(\Omega)$, we have

$$
\left\|\nabla \widehat{u}_{n}\right\|_{p}^{p} \longrightarrow\|\nabla \widehat{u}\|_{p}^{p}
$$

From (3.29), (3.32) and (3.33), we see that

$$
\left\|\nabla \widehat{u}_{n}\right\|_{p}^{p}-\int_{\left\{\widehat{u}_{n} \neq 0\right\}} \eta_{0} \widehat{u}_{n}^{p} d z<0 \text { for large } n \geqslant 1 \text {. }
$$

This means that we can find $u \in W^{1, p}(\Omega) \cap L^{\infty}(\Omega)$, such that

$$
\|\nabla u\|_{p}^{p}-\int_{\{u \neq 0\}} \eta_{0} u^{p} d z<0, \quad u \geqslant 0 .
$$

Moreover, dividing with $\|u\|_{p}^{p}$ if necessary, we may assume that $\|u\|_{p}=1$. For $\zeta>0$, we have

$$
F(z, \zeta)=\int_{0}^{1} \frac{d}{d t} F(z, t \zeta) d t=\int_{0}^{1} f(z, t \zeta) \zeta d t
$$


so, using hypothesis $H_{f}$ (ii), we have

$$
\frac{F(z, \zeta)}{\zeta^{p}}=\int_{0}^{1} \frac{f(z, t \zeta)}{\zeta^{p-1}} d t \geqslant \frac{f(z, \zeta)}{\zeta^{p-1}} \int_{0}^{1} t^{p-1} d t=\frac{1}{p} \frac{f(z, \zeta)}{\zeta^{p-1}}
$$

and thus

$$
\liminf _{\zeta \rightarrow 0^{+}} \frac{F(z, \zeta)}{\zeta^{p}} \geqslant \frac{1}{p} \eta_{0}(z) \text { for almost all } z \in \Omega
$$

Consider $u \in W^{1, p}(\Omega) \cap L^{\infty}(\Omega),\|u\|_{p}=1$ satisfying (3.34). For $r \in(0,1)$ small, we will have $r u(z) \in[0,1]$ for almost all $z \in \Omega$. Then, using hypothesis $H_{f}$ (iii), we have

$$
\begin{aligned}
\frac{F(z, r u(z))}{r^{p}} & =\frac{1}{r^{p}} \int_{0}^{r u(z)} f(z, s) d s \geqslant-\frac{1}{r^{p}}\|f(\cdot, 1)\|_{\infty} \int_{0}^{r u(z)} s^{p-1} d s \\
& \geqslant-\frac{\|f(\cdot, 1)\|_{\infty}}{p} u(z)^{p} \geqslant-\frac{\|f(\cdot, 1)\|_{\infty}}{p}\|u\|_{\infty}^{p} .
\end{aligned}
$$

From (3.35), (3.36) and Fatou's lemma, we have

$$
\liminf _{r \rightarrow 0^{+}} \int_{\{u \neq 0\}} \frac{F(z, r u)}{r^{p}} d z \geqslant \frac{1}{p} \int_{\{u \neq 0\}} \eta_{0} u^{p} d z
$$

so, using also (3.34), we have

$$
\frac{1}{p}\|\nabla u\|_{p}^{p}-\int_{\Omega} \frac{F(z, r u)}{r^{p}} d z<0 \text { for small } r \in(0,1),
$$

thus

$$
\widehat{\varphi}(r u)<0 \text { for small } r \in(0,1)
$$

(recall that $r u \geqslant 0$ and see (3.1)). Using also (3.28), we see that

$$
\widehat{m}=\widehat{\varphi}\left(u_{0}\right)<0=\widehat{\varphi}(0)
$$

and so $u_{0} \neq 0$.

This completes the proof of Claim 1 .

Claim 2. $u_{0} \in L^{\infty}(\Omega)$ 
For $k \geqslant 1$, we introduce the truncation

$$
f_{k}(z, \zeta)= \begin{cases}f(z, 0) & \text { if } \zeta \leqslant 0 \\ \max \left\{f(z, \zeta),-k \zeta^{p-1}\right\} & \text { if } \zeta>0\end{cases}
$$

Evidently this is a Carathéodory function, $f_{k}(\cdot, \zeta) \in L^{\infty}(\Omega)$ for all $\zeta \in \mathbb{R}$ and

$$
\left|f_{k}(z, \zeta)\right| \leqslant c_{4}\left(1+|\zeta|^{p-1}\right) \text { for almost all } z \in \Omega \text {, all } \zeta \in \mathbb{R}
$$

for some $c_{4}>0$. We set

$$
\eta_{0}^{k}(z)=\liminf _{\zeta \rightarrow 0^{+}} \frac{f_{k}(z, \zeta)}{\zeta^{p-1}} \text { and } \eta^{k}(z)=\limsup _{\zeta \rightarrow+\infty} \frac{f_{k}(z, \zeta)}{\zeta^{p-1}}
$$

Since

$$
f_{k}(z, \zeta) \geqslant f(z, \zeta) \text { for almost all } z \in \Omega \text {, all } \zeta \in \mathbb{R}
$$

we see that $\eta_{0}^{k} \geqslant \eta_{0}$ and so

$$
\widehat{\lambda}_{1}\left(-\eta_{0}^{k}\right) \leqslant \widehat{\lambda}_{1}\left(-\eta_{0}\right)<0 \quad \forall k \geqslant 1
$$

(see (2.6) and hypothesis $H_{f}$ (iv)).

Moreover, from (3.37), we see that $\eta^{k} \searrow \eta$ and so

$$
\widehat{\lambda}_{1}\left(-\eta^{k}\right) \longrightarrow \widehat{\lambda}_{1}(-\eta)>0
$$

(see (2.6) and hypothesis $H_{f}$ (iii)). Hence, we have

$$
\widehat{\lambda}_{1}\left(-\eta^{k}\right)>0 \text { for large } k \geqslant 1 \text {. }
$$

Reasoning as before, we obtain $u_{0 k} \in W^{1, p}(\Omega), u_{0 k} \geqslant 0, u_{0 k} \neq 0$ which minimizes $\widehat{\varphi}_{k}$ (here $\widehat{\varphi}_{k}$ is defined as $\widehat{\varphi}$ with $f(z, \zeta)$ replaced by $f_{k}(z, \zeta)$ ). Note that because of (3.38), we have $\widehat{\varphi}_{k} \in C^{1}\left(W^{1, p}(\Omega)\right)$ and so for $k \geqslant 1$ large, we have

$$
\widehat{\varphi}_{k}^{\prime}\left(u_{0 k}\right)=0
$$

so

$$
A\left(u_{0 k}\right)=N_{f_{k}}\left(u_{0 k}\right)
$$

with $N_{f_{k}}(u)(\cdot)=f_{k}(\cdot, u(\cdot))$ for all $u \in W^{1, p}(\Omega)$ (recall that $u_{0 k} \geqslant 0$ ). Thus

$$
\left\{\begin{array}{l}
-\Delta_{p} u_{0 k}(z)=f_{k}\left(z, u_{0 k}(z)\right) \text { in } \Omega \\
\frac{\partial u_{0 k}}{\partial n}=0 \text { on } \partial \Omega
\end{array}\right.
$$


Nonlinear regularity theory implies that $u_{0 k} \in C^{1}(\bar{\Omega})$ for all $k \geqslant 1$ (see Lieberman [21] and Gasiński and Papageorgiou [8, pp. 738-739]). We set

$$
v_{k}=\min \left\{u_{0}, u_{0 k}\right\} \in W^{1, p}(\Omega) \cap L^{\infty}(\Omega) \quad \forall k \geqslant 1 .
$$

Since $u_{0 k}$ is a minimizer of $\widehat{\varphi}_{k}$, we have

$$
\widehat{\varphi}_{k}\left(u_{0 k}\right) \leqslant \widehat{\varphi}_{k}(h) \quad \forall h \in W^{1, p}(\Omega) .
$$

So, if we choose $h=\max \left\{u_{0}, u_{0 k}\right\} \in W^{1, p}(\Omega)$, then

$$
\begin{aligned}
& \frac{1}{p} \int_{\left\{u_{0 k}<u_{0}\right\}}\left\|\nabla u_{0 k}\right\|^{p} d z-\int_{\left\{u_{0 k}<u_{0}\right\}} F_{k}\left(z, u_{0 k}\right) d z \\
& \leqslant \frac{1}{p} \int_{\left\{u_{0 k}<u_{0}\right\}}\left\|\nabla u_{0}\right\|^{p} d z-\int_{\left\{u_{0 k}<u_{0}\right\}} F_{k}\left(z, u_{0}\right) d z,
\end{aligned}
$$

so

$$
\begin{aligned}
& \frac{1}{p} \int_{\left\{u_{0 k}<u_{0}\right\}}\left(\left\|\nabla u_{0 k}\right\|^{p}-\left\|\nabla u_{0}\right\|^{p}\right) d z \\
& \leqslant \int_{\left\{u_{0 k}<u_{0}\right\}}\left(F_{k}\left(z, u_{0 k}\right)-F_{k}\left(z, u_{0}\right)\right) d z .
\end{aligned}
$$

We have

$$
\begin{aligned}
\widehat{\varphi}\left(v_{k}\right)-\widehat{\varphi}\left(u_{0}\right)= & \frac{1}{p} \int_{\left\{u_{0 k}<u_{0}\right\}}\left(\left\|\nabla u_{0 k}\right\|^{p}-\left\|\nabla u_{0}\right\|^{p}\right) d z \\
& -\int_{\left\{u_{0 k}<u_{0}\right\}}\left(F\left(z, u_{0 k}\right)-F\left(z, u_{0}\right)\right) d z \\
\leqslant & \int_{\left\{u_{0 k}<u_{0}\right\}}\left(F_{k}\left(z, u_{0 k}\right)-F_{k}\left(z, u_{0}\right)-F\left(z, u_{0 k}\right)-F\left(z, u_{0}\right)\right) d z
\end{aligned}
$$

(see (3.39)).

But on $\left\{u_{0 k}<u_{0}\right\}$, we have

$$
F_{k}\left(z, u_{0 k}\right)-F_{k}\left(z, u_{0}\right)-F\left(z, u_{0 k}\right)+F\left(z, u_{0}\right)=\int_{u_{0 k}}^{u_{0}}\left(-f_{k}(z, s)+f(z, s)\right) d z \leqslant 0
$$


(recall that $\left.f_{k} \geqslant f\right)$. Using (3.41) in (3.40), we obtain

$$
\widehat{\varphi}\left(v_{k}\right) \leqslant \widehat{\varphi}\left(u_{0}\right)=\widehat{m},
$$

so

$$
\widehat{\varphi}\left(v_{k}\right)=\widehat{m}
$$

Since $v_{k} \in L^{\infty}(\Omega)$, we conclude that Claim 2 holds.

Next, let $h \in C^{1}(\bar{\Omega})$ and $t \in(-1,1)$. We set

$$
w(h)=\int_{\Omega}\left(G\left(z, u_{0}+t h\right)-G\left(z, u_{0}\right)-g\left(z, u_{0}\right) h\right) d z,
$$

so

$$
\begin{aligned}
|w(h)| & \leqslant \int_{\Omega} \int_{0}^{1}\left|g\left(z, u_{0}+t h\right)-g\left(z, u_{0}\right)\right| d t|h| d z \\
& \leqslant \int_{0}^{1}\left\|N_{g}\left(u_{0}+t h\right)-N_{g}\left(u_{0}\right)\right\|_{p^{\prime}} d t\|h\|,
\end{aligned}
$$

where $N_{g}(u)(\cdot)=g(\cdot, u(\cdot))$ for all $u \in W^{1, p}(\Omega)$. Here we have used Fubini's theorem and Hölder's inequality. Hypotheses $H_{f}$ (i), (ii) and the fact that $h \in C^{1}(\bar{\Omega})$, imply that

$$
\left|g\left(z, u_{0}(z)+t h(z)\right)\right| \leqslant \widehat{a}(z) \text { for almost all } z \in \Omega \text {, all } t \in(-1,1),
$$

with $\widehat{a} \in L^{\infty}(\Omega)$. From this it follows that

$$
\int_{0}^{1}\left\|N_{g}\left(u_{0}+t h\right)-N_{g}\left(u_{0}\right)\right\|_{p} d t \longrightarrow 0 \quad \text { as }\|h\| \rightarrow 0 .
$$

Therefore

$$
\frac{|w(h)|}{\|h\|} \longrightarrow 0 \quad \text { as }\|h\| \rightarrow 0
$$

and so we see that the Gâteaux derivative exists at $u_{0}$ in every direction $h \in C^{1}(\bar{\Omega})$ and is equal to $A\left(u_{0}\right)-N_{f}\left(u_{0}\right)$ (recall that $u_{0} \geqslant 0$ and see (3.1)). Moreover, by virtue of (3.28), we have

$$
\left\langle\widehat{\varphi}_{G}^{\prime}\left(u_{0}\right), h\right\rangle=\left\langle A\left(u_{0}\right)-N_{g}\left(u_{0}\right), h\right\rangle=0 \quad \forall h \in C^{1}(\bar{\Omega}) .
$$


Since the embedding $C^{1}(\bar{\Omega}) \subseteq W^{1, p}(\Omega)$ is dense, it follows that

$$
A\left(u_{0}\right)=N_{f}\left(u_{0}\right)
$$

so

$$
\left\{\begin{array}{l}
-\Delta_{p} u_{0}(z)=f\left(z, u_{0}(z)\right) \text { in } \Omega \\
\frac{\partial u_{0}}{\partial n}=0 \text { on } \partial \Omega
\end{array}\right.
$$

(see Motreanu and Papageorgiou [22]).

Note that $f\left(\cdot, u_{0}(\cdot)\right) \in L^{\infty}(\Omega)$ (see hypothesis $H_{f}(\mathrm{i})$ and recall that by Claim 2, $u_{0} \in L^{\infty}(\Omega)$ ). So, from nonlinear regularity theory, we have that $u_{0} \in C^{1}(\bar{\Omega})$. By virtue of hypothesis $H_{f}$ (ii), we have

$$
\frac{f\left(z, u_{0}(z)\right)}{u_{0}(z)^{p-1}} \geqslant \frac{f\left(z,\left\|u_{0}\right\|_{\infty}\right)}{\left\|u_{0}\right\|_{\infty}^{p-1}} \text { for almost all } z \in\left\{u_{0}>0\right\},
$$

so

$$
f\left(z, u_{0}(z)\right) \geqslant \frac{-\left\|f\left(\cdot,\left\|u_{0}\right\|_{\infty}\right)\right\|_{\infty}}{\left\|u_{0}\right\|_{\infty}^{p-1}} u_{0}(z)^{p-1} \text { for almost all } z \in\left\{u_{0}>0\right\}
$$

Also, recall that $f(z, 0) \geqslant 0$ for almost all $z \in \Omega$. Therefore from (3.42), it follows that

$$
\Delta_{p} u_{0}(z) \leqslant c_{5} u_{0}(z)^{p-1} \text { for almost all } z \in \Omega,
$$

with $c_{5}>0$ and so

$$
u_{0}(z)>0 \quad \forall z \in \bar{\Omega}
$$

(see Vázquez [25]).

\section{Uniqueness of positive solutions}

Next we show the uniqueness of positive solutions for problem (1.1). In fact, we show that hypotheses $H_{f}$ (iii) and (iv) are both necessary and sufficient for the existence and uniqueness of positive solutions for problem (1.1).

Proposition 4.1 If hypotheses $H_{f}$ hold, then problem (1.1) has a unique positive solution $u_{0} \in C^{1}(\bar{\Omega})$, such that $u_{0}(z)>0$ for all $z \in \bar{\Omega}$.

Proof Let $u, v$ be two positive solutions for problem (1.1). Then we have $u, v \in$ $W^{1, p}(\Omega) \cap L^{\infty}(\Omega)$ (see Motreanu and Papageorgiou [22]) and moreover, as before 
through nonlinear regularity theory and the nonlinear maximum principle of Vázquez [25], we have that $u(z)>0$ and $v(z)>0$ for all $z \in \bar{\Omega}$. Let

$$
R(u, v)(z)=\|\nabla u(z)\|^{p}-\|\nabla v(z)\|^{p-2}\left(\nabla v(z), \nabla\left(\frac{u(z)^{p}}{v(z)^{p-1}}\right)\right)_{\mathbb{R}^{N}} .
$$

From Allegretto and Huang [1], we know that

$$
R(u, v)(z) \geqslant 0 \quad \forall z \in \bar{\Omega} .
$$

Using the nonlinear Green's identity (see Casas and Fernández [5]), we have

$$
\begin{aligned}
\int_{\Omega} \frac{f(z, u)}{u^{p-1}}\left(u^{p}-v^{p}\right) d z & =-\int_{\Omega} \Delta_{p} u\left(u-\frac{v^{p}}{u^{p-1}}\right) d z \\
& =\int_{\Omega}\|\nabla u\|^{p-2}\left(\nabla u, \nabla u-\nabla\left(\frac{v^{p}}{u^{p-1}}\right)\right)_{\mathbb{R}^{N}} d z \\
& =\|\nabla u\|_{p}^{p}-\int_{\Omega}\|\nabla u\|^{p-2}\left(\nabla u, \nabla\left(\frac{v^{p}}{u^{p-1}}\right)\right)_{\mathbb{R}^{N}} d z \\
& =\|\nabla u\|_{p}^{p}-\|\nabla v\|_{p}^{p}+\int_{\Omega} R(v, u) d z .
\end{aligned}
$$

Similarly, interchanging the roles of $u$ and $v$, we also have

$$
\int_{\Omega} \frac{f(z, v)}{v^{p-1}}\left(v^{p}-u^{p}\right) d z=\|\nabla v\|_{p}^{p}-\|\nabla u\|_{p}^{p}+\int_{\Omega} R(u, v) d z .
$$

Adding (4.2) and (4.3), using hypothesis $H_{f}$ (ii) and recalling that $R \geqslant 0$, we obtain $0 \geqslant \int_{\Omega}\left(\frac{f(z, u)}{u^{p-1}}-\frac{f(z, v)}{v^{p-1}}\right)\left(u^{p}-v^{p}\right) d z=\int_{\Omega}(R(v, u)+R(u, v)) d z \geqslant 0$,

so

$$
\int_{\Omega}(R(v, u)+R(u, v)) d z=0
$$

and thus

$$
R(v, u)=R(u, v)=0 \text { for almost all } z \in \Omega,
$$

thus

$$
u=k v
$$


for some $k>0$ (see Allegretto and Huang [1]). Hypothesis $H_{f}$ (ii) implies that $k=1$ and so $u=v$.

As we already remarked, we are going to show that hypotheses $H_{f}$ (iii) and (iv) are also necessary for the uniqueness of positive solutions for problem (1.1).

Proposition 4.2 If $f: \Omega \times \mathbb{R} \longrightarrow \mathbb{R}$ is a Carathéodory function satisfying hypotheses $H_{f}$ (i) and (ii) and problem (1.1) has a unique positive solution $u_{0} \in W^{1, p}(\Omega)$, then $\widehat{\lambda}_{1}\left(-\eta_{0}\right)<0<\widehat{\lambda}_{1}(-\eta)$, where

$$
\eta_{0}(z)=\lim _{\zeta \rightarrow 0^{+}} \frac{f(z, \zeta)}{\zeta^{p-1}} \text { and } \eta(z)=\lim _{\zeta \rightarrow+\infty} \frac{f(z, \zeta)}{\zeta^{p-1}}
$$

Proof Note that $u_{0} \in W^{1, p}(\Omega) \cap L^{\infty}(\Omega)$ (see e.g., Hu and Papageorgiou [17]) and as before via nonlinear regularity (see Lieberman [21]) and the nonlinear maximal principle (see Vázquez [25]), we have $u_{0} \in C^{1}(\bar{\Omega})$ with $u_{0}(z)>0$ for all $z \in \bar{\Omega}$.

Using (2.6) and hypothesis $H_{f}$ (ii), we have

$$
\begin{aligned}
\widehat{\lambda}_{1}\left(-\eta_{0}\right) & \leqslant \frac{\left\|\nabla u_{0}\right\|_{p}^{p}-\int_{\Omega} \eta_{0} u_{0}^{p} d z}{\left\|u_{0}\right\|_{p}^{p}} \\
& =\frac{\int_{\Omega} f\left(z, u_{0}\right) u_{0} d z-\int_{\Omega} \eta_{0} u_{0}^{p} d z}{\left\|u_{0}\right\|_{p}^{p}} \\
& <\frac{\int_{\Omega} \eta_{0} u_{0}^{p} d z-\int_{\Omega} \eta_{0} u_{0}^{p} d z}{\left\|u_{0}\right\|_{p}^{p}} \\
& =0 .
\end{aligned}
$$

This proves that $\widehat{\lambda}_{1}\left(-\eta_{0}\right)<0$.

Next, let

$$
\beta(z)=-\frac{f\left(z,\left\|u_{0}\right\|_{\infty}+1\right)}{\left(\left\|u_{0}\right\|_{\infty}+1\right)^{p-1}}
$$

Then $\beta \in L^{\infty}(\Omega)$. By virtue of Proposition 2.1, problem (2.1) with this particular weight $\beta$, has a principal eigenfunction $\widehat{u}_{1} \in C^{1}(\bar{\Omega})$, such that $\widehat{u}_{1}(z)>0$ for all $z \in \bar{\Omega}$. Let $k>0$ be large enough, such that $u_{0}<k \widehat{u}_{1}=\widetilde{u}_{1}$. As before (see the proof of Proposition 4.1), we have

$$
\int_{\Omega} \frac{f\left(z, u_{0}\right)}{u_{0}^{p-1}}\left(u_{0}^{p}-\widetilde{u}_{1}^{p}\right) d z=\left\|\nabla u_{0}\right\|_{p}^{p}-\left\|\nabla \widetilde{u}_{1}\right\|_{p}^{p}+\int_{\Omega} R\left(\widetilde{u}_{1}, u_{0}\right) d z
$$

and

$$
\int_{\Omega}\left(\widehat{\lambda}_{1}(\beta)-\beta\right)\left(\widetilde{u}_{1}^{p}-u_{0}^{p}\right) d z=\left\|\nabla \widetilde{u}_{1}\right\|_{p}^{p}-\left\|\nabla u_{0}\right\|_{p}^{p}+\int_{\Omega} R\left(u_{0}, \widetilde{u}_{1}\right) d z .
$$


Adding (4.4) and (4.5), we obtain

$$
\int_{\Omega}\left(\frac{f\left(z, u_{0}\right)}{u_{0}^{p-1}}+\beta-\widehat{\lambda}_{1}(\beta)\right)\left(u_{0}^{p}-\widetilde{u}_{1}^{p}\right) d z=\int_{\Omega}\left(R\left(\widetilde{u}_{1}, u_{0}\right)+R\left(u_{0}, \widetilde{u}_{1}\right)\right) d z \geqslant 0 .
$$

Note that by virtue of hypothesis $H_{f}$ (ii), we have

$$
\frac{f\left(z, u_{0}\right)}{u_{0}^{p-1}}>\frac{f\left(z,\left\|u_{0}\right\|_{\infty}+1\right)}{\left(\left\|u_{0}\right\|_{\infty}+1\right)^{p-1}}=-\beta(z) \text { for almost all } z \in \Omega
$$

so

$$
\frac{f\left(z, u_{0}\right)}{u_{0}^{p-1}}+\beta(z)>0 \text { for almost all } z \in \Omega
$$

Also, recall that

$$
\left(u_{0}^{p}-\tilde{u}_{1}^{p}\right)(z)<0 \text { for almost all } z \in \Omega .
$$

So, using (4.7) and (4.8) in (4.6), we infer that

$$
\widehat{\lambda}_{1}(\beta)>0 .
$$

But $\beta \leqslant-\eta$ (see hypothesis $H_{f}$ (ii)) and so $\widehat{\lambda}_{1}(\beta) \leqslant \widehat{\lambda}_{1}(-\eta)$. Hence $\widehat{\lambda}_{1}(-\eta)>0$.

So, summarizing the situation for problem (1.1), we can state the following theorem.

Theorem 4.3 If $f: \Omega \times \mathbb{R} \longrightarrow \mathbb{R}$ is a Carathéodory function which satisfies hypotheses $H_{f}(\mathrm{i})$ and (ii), then problem (1.1) admits a unique positive solution if and only if

$$
\widehat{\lambda}_{1}\left(-\eta_{0}\right)<0<\widehat{\lambda}_{1}(\eta)
$$

where

$$
\eta_{0}(z)=\lim _{\zeta \rightarrow 0^{+}} \frac{f(z, \zeta)}{\zeta^{p-1}} \text { and } \eta(z)=\lim _{\zeta \rightarrow+\infty} \frac{f(z, \zeta)}{\zeta^{p-1}}
$$

Acknowledgments The authors wish to thank the referee for his/her remarks and for bringing to their attention Reference [3].

Open Access This article is distributed under the terms of the Creative Commons Attribution License which permits any use, distribution, and reproduction in any medium, provided the original author(s) and the source are credited. 


\section{References}

1. Allegretto, W., Huang, Y.-X.: A Picone's identity for the $p$-Laplacian and applications. Nonlinear Anal. 32, 819-830 (1998)

2. Amann, H.: On the existence of positive solutions of nonlinear elliptic boundary value problems. Indiana Univ. Math. J. 21, 125-146 (1971)

3. Belloni, M., Kawohl, B.: A direct uniqueness proof for equations involving the $p$-Laplacian operator. Manuscr. Math. 109, 229-231 (2002)

4. Brézis, H., Oswald, L.: Remarks on sublinear elliptic equations. Nonlinear Anal. 10, 55-64 (1986)

5. Casas, E., Fernández, L.A.: A Green's formula for quasilinear elliptic operators. J. Math. Anal. Appl. 142, 62-73 (1989)

6. Dancer, N.: Uniqueness for elliptic equations when a parameter is large. Nonlinear Anal. 8, 835-836 (1984)

7. de Figueiredo, D.G.: Differential Equations (San Paulo, 1981), Positive Solutions of Semilinear Elliptic Problems. Lecture Notes in Mathematics, vol. 957, pp. 34-85. Springer, New York (1982)

8. Gasiński, L., Papageorgiou, N.S.: Nonlinear Analysis. Chapman and Hall/CRC Press, Boca Raton (2006)

9. Gasiński, L., Papageorgiou, N.S.: Existence and multiplicity of solutions for Neumann $p$-Laplaciantype equations. Adv. Nonlinear Stud. 8, 843-870 (2008)

10. Gasiński, L., Papageorgiou, N.S.: Nontrivial solutions for a class of resonant $p$-Laplacian Neumann problems. Nonlinear Anal. 71, 6365-6372 (2009)

11. Gasiński, L., Papageorgiou, N.S.: Nontrivial solutions for Neumann problems with an indefinite linear part. Appl. Math. Comput. 217, 2666-2675 (2010)

12. Gasiński, L., Papageorgiou, N.S.: Existence of three nontrivial smooth solutions for nonlinear resonant neumann problems driven by the $p$-Laplacian. J. Anal. Appl. 29, 413-428 (2010)

13. Gasiński, L., Papageorgiou, N.S.: Multiple solutions for nonlinear Neumann problems with asymmetric reaction, via Morse theory. Adv. Nonlinear Stud. 11, 781-808 (2011)

14. Guo, Z.: On the number of positive solutions for quasilinear elliptic problems. Nonlinear Anal. 27, 229-247 (1996)

15. Guo, Z., Webb, J.R.L.: Uniqueness of positive solutions for quasilinear elliptic equations when a parameter is large. Proc. Roy. Soc. Edinburgh Sect. A 124, 189-198 (1994)

16. Hess, P.: On uniqueness of positive solutions of nonlinear elliptic boundary value problems. Math. Z 154, 17-18 (1977)

17. Hu, S., Papageorgiou, N.S.: Nonlinear Neumann equations driven by a nonhomogeneous differential operator. Commun. Pure Appl. Math. 9, 1801-1827 (2010)

18. Kamin, S., Veron, L.: Flat core properties associated to the $p$-Laplace operator. Proc. Am. Math. Soc. 118, 1079-1085 (1993)

19. Krasnoselskii, M.A.: Positive Solutions of Operator Equations. Noordhoff International Publishing, Groningen (1964)

20. Laetsch, T.: Uniqueness for sublinear boundary value problems. J. Diff. Equ. 13, 13-23 (1973)

21. Lieberman, G.M.: Boundary regularity for solutions of degenerate elliptic equations. Nonlinear Anal. 12, 1203-1219 (1988)

22. Motreanu, D., Papageorgiou, N.S.: Existence and multiplicity of solutions for Neumann problems. J. Diff. Equ. 232, 1-35 (2007)

23. Papageorgiou, N.S., Kyritsi, S.: Handbook of Applied Analysis. Springer, New York (2009)

24. Simpson, R.B., Cohen, D.S.: Positive solutions of nonlinear elliptic eigenvalue problem. J. Math. Mech. 19, 895-910 (1969/1970)

25. Vázquez, J.L.: A strong maximum principle for some quasilinear elliptic equations. Appl. Math. Optim. 12, 191-202 (1984) 